DOI: 10.12731/2227-930X-2017-1-52-64

\title{
ALGORITHMS FOR TRAFFIC MANAGEMENT IN THE INTELLIGENT TRANSPORT SYSTEMS
}

\author{
Nikolaev A.B., Aung Myo Thiwn, Myo Lin Aung, \\ Myo Min Khaing, Aung Nyi Nyi Zaw
}

Traffic jams interfere with the drivers and cost billions of dollars per year and lead to a substantial increase in fuel consumption. In order to avoid such problems the paper describes the algorithms for traffic management in intelligent transportation system, which collects traffic information in real time and is able to detect and manage congestion on the basis of this information. The results show that the proposed algorithms reduce the average travel time, emissions and fuel consumption. In particular, travel time has decreased by about $23 \%$, the average fuel consumption of $9 \%$, and the average emission of $10 \%$.

Keywords: traffic jams; intelligent transport systems; detection and control of congested roads; automated control system; control; simulation; algorithm.

\section{Introduction}

Traffic jams are a major problem in large urban centers and directly impact on the economy due to increase fuel consumption. In addition, it affects the productivity of society as congestion dramatically increase travel time from one point to another and causing irreparable damage to the environment due to the increase of the emission of polluting gases. According to statistics, $85 \%$ is associated with time wasted in traffic, $13 \%$ due to the increase in fuel consumption and $2 \%$ can be attributed to an increase in emissions of polluting gases [1, 2].

To prevent congestion and improve the efficiency of transport systems, there is an active implementation of intelligent transport systems (ITS). Intelligent transport systems use advances in techonology in the area of processing, sensing and communication in order to monitor 
traffic conditions in a particular region to manage traffic and reduce congestion as well as reduce the number of road accidents. In addition, they can be used to provide information and entertainment services to drivers and passengers in order to make their journey a more pleasant process $[2,3]$. A Vehicular Network is an important component in an ITS. In this network, vehicles are equipped with processors, sensors and wireless communication interfaces so that they can communicate with one another and with the elements in the network infrastructure (RSU - Road Side Unit), thus creating an ad hoc network while vehicles move through roads. Thus, when driving vehicles on the roads equipped with ITS, it created a special network $(4,7,10)$.

Some researchers $[8,9]$ proposed the architecture for the design of ITS, which range from centralized solutions to distributed decision making solutions. These solutions use both the information provided by vehicles and the characteristics of the infrastructure of the roads to detect and control congestion. Thus, after detecting a congested area or road, a rerouting mechanism is applied to the vehicles to prevent more vehicles from entering the affected area, which results in a more efficient traffic. Thus, this work is aimed at the implementation of a distributed ITS to detect congestion and traffic management.

The proposed system consists of a set of roadside controllers. Each controller is responsible for roadside vehicles management and congestion detection only within the coverage area of its range. Moreover, the system includes a congestion control mechanism that periodically performs routing of all vehicles so they do not pass through congested areas. The system is divided into four stages, the distribution of the roadside unit RSU collection and transmission of data, congestion detection and congestion control [5].

\section{The algorithm of placing of roadside controllers (RSU) on the map}

Allocation of road side controller (RSU) is uniform depending on their radius and size of the map. Thus, the larger the range, the smaller the number of roadside controllers is used to cover the map. In al- 
gorithm 1, when placing roadside controllers are taken into account: Width - the total width of the card, Length - total length of the card and Radius - range roadside communication controller. Algorithm 1 based on the size of geographic area and radius roadside communication controller (line 3 ) calculates the number of roadside controllers that are needed to cover the entire map. Then the coordinates of the placement of roadside controllers in relation to the map.

The algorithm of placing of roadside controllers (RSU) on the map Input: Width // Total width of the map

1 Length // Total length of the map

2 Radius // Operating range of RSU

Output: Coordinates of the points of each RSU

// returns the amount of RSUS to cover the entire map

3 rsus $\leftarrow$ getNumberOfRSUs(Width; Length; Radius);

4 foreach $\gamma \in$ rsus do

// returns the coordinates (x; y) for each RSU

5 Rsus_coordinates.add(getCoordinates(r));

6 end

Communication range of roadside controllers influences the cost of implementing the system, i.e, the smaller the radius of the connection, the greater the number of roadside controllers required to cover the entire map.

\section{Algorithm to collect and transmit data in the RSU}

This algorithm provides data collection from RSU to obtain information about the events taking place within their communication range, as well as information about the behavior of the vehicle in their coverage area. In which vehicles periodically send information about the position, speed, distance covered, time and direction of travel movement in the near RSU. This information is transmitted via the long-range communication (LTE or $3 \mathrm{G}$ ).

Algorithm 2 describes a process in which each vehicle sends the information to the nearest RSU. The algorithm takes as input the set of vehicles and a set of coordinates of each RSU, which are respective- 
ly represented N and RSUs-Coordinates. Thus, each vehicle collects information and identifies the nearest RSU in relation to its position (Line 4). Moreover, each vehicles create messages containing the collected information and sends it to the nearest RSU (Lines 5-6). After collecting and transmitting data to the RSU, it is necessary its analysis and interpretation. Each roadside controller creates a graph $\mathrm{G}=(\mathrm{V}, \mathrm{A})$, where the vertices $\mathrm{V}$ - a set of transitions within the reach of roadside controller (representing the top) and the $\operatorname{arc} \mathrm{A}$ - is a set of roads that connect the top. Each road (edge) of graph $\mathrm{G}$ has a weight defined by the average speed with which the vehicles travel it and the maximum speed allowed on it. This weight is inversely proportional to the speed at which the vehicle moves along the road. This weight is inversely proportional to the speed at which the vehicle moves along the road. Thus, the closer to the speed of movement of the vehicle with speed limited, the lower the weight of the road. Conversely, the lower the speed of the vehicle relative to the maximum speed, the higher the weight.

Алгоритм of Collecting and sending data to the nearest RSU Input: $\mathrm{N} / /$ Set of vehicles on the network

1 RSUs_Coordinates // Set with the coordinates of each RSU

2 foreach $v \in N$ do

// returns information about vehicle $\mathrm{V}$

3 status v.getInformations();

// returns the nearest RSU to vehicleV

4 nearestRsu RSUs_Coordinates.getNearstRSU(v.getPosition()); $/ /$ creates a message with the information of vehicle $\mathrm{v}$ and sends it to the nearest RSU

$5 \quad$ message $\leftarrow$ CreateMessage(status);

6 message.sendTo(nearestRsu);

7 end

\section{The algorithm of alternative routing of vehicle}

The change of route of vehicle movement occurs periodically depending on the current traffic. Each roadside controller is responsible 
for the selection of a new route within its range. The distortion depends on the intensity of vehicle movement and the weight of the road. When an overload vehicle are forwarded to another route to avoid congested areas. As the base routing algorithm is used to find the shortest route in time. However, the set of $\mathrm{K}$ shortest paths is calculated as alternative ways in which $\mathrm{K}$ can be easily adjusted to adapt better to the behavior in the network. Of these alternative routes, the only way is selected by using the probability distribution of Boltzmann.

Algorithm 3 describes the process of alternative routing of the vehicle. The algorithm takes as input a set of vehicles within a radius of communication of each RSU (N). The graph is created with the appropriate functions described previously (represented in the algorithm as $\mathrm{G}$, and the variable $\mathrm{K}$, which represents the number of shortest routes that must be calculated). Thus, the RSU computes the path of each vehicle and its current coordinates (lines 4-5). Then, the RSU calculates the destination vehicle $\mathrm{V}$ is located within its coverage area (the last part of the vehicle ways within the RSU). After the detection of the last section of the path within the RSU, $\mathrm{k}$ shortest paths are calculated starting from the current point to the final vehicle In the path (line 7). From the K shortest past, a new path is selected based on the probability distribution of Boltzmann (line 8).

Algorithm of alternative routing of vehicles

input: $\mathrm{N} / /$ a Set of vehicles in range of each RSU

$1 \quad \mathrm{G} / /$ the Graph is created for each RSU

$2 \mathrm{~K} / /$ Number of alternative ways

$3 \quad$ For $v \in N$ do

// sets of edges that make up the route of vehicles $\mathrm{V}$

4 route v.getRoute();

// returns the current coordinates of the vehicle and the distance to the last segment of the path $\mathrm{V}$, contained in the graph $\mathrm{G}$ for a given $\mathrm{RSU}$ $5 \quad$ source $\leftarrow$ v.getPosition();

$6 \quad$ lastEdge $\leftarrow$ G.getLastEdge(route);

// calculates $\mathrm{K}$ shortest paths from the starting point to the last point of movement of the vehicle 
$7 \quad$ alternativeRoutes $\leftarrow$ G.getKShortestPaths (source; lastEdge; $K$ ); // select a path from a set of alternative paths

8 newRoute $\leftarrow$ boltzmann(alternativeRoutes; $\mathrm{G}$ );

9 if lastEdge! = route.getDestination() then

// returns the remaining part of the route of vehicle $\mathrm{V}$ from the last section

10 remaningEdges $\leftarrow$ route.split(lastEdge);

11 newRoute.add(remaningEdges);

12 v.setRoute (newRoute);

13 end

14 else

15 v.setRoute(newRoute);

16 end

17 end

Select the path among $K$ shortest routes is based on the calculation of weights, defined as the sum of the weights of all segments of the path included in the route. For load balancing across shortest routes uses a lot of $R_{j}$. For route determination uses the following rules:

$J=$ set of vehicles on the map

$W j=$ set of paths of the vehicle $j(j \in j)$

$\gamma_{J}^{I}=$ path $i$ of vehicle $\mathrm{j}(j \in j)$ and $\left(\gamma_{J}^{I} \in R_{j}\right)$

$\omega_{J}^{I}=$ weight of path $\gamma_{J}^{I}$

$M\left(\omega_{J}^{I}\right)=$ normalized value of $\omega_{J}^{I}\left(\omega_{j}^{i} \in[0,1]\right)$ defined by Equation. 1:

$$
M\left(\omega_{J}^{I}\right)=\frac{P\left(\gamma_{J}^{I}\right)}{\max \left\{R\left(\gamma_{J}^{i}\right) \mid \forall \in W j\right\}}
$$

The $H_{J}^{I}$ is the Boltzmann constant of vehicle $j$ for temperature $T$, according to equation 2 :

$$
H_{j}^{i}=\sum_{i \in R_{j}} e^{-\left(U\left(\omega_{j}^{i}\right) / T\right)}
$$

$P_{J}^{I}$ is the probability of choosing path $i$ of vehicle $j$ with the parameter of temperature $\mathrm{T}$, 


$$
P_{T}^{j}\left(\gamma_{j}^{i}\right)=\frac{1}{K_{N}^{j}} e^{-\left(U\left(\omega_{j}^{i}\right) / T\right)}
$$

where: $T \longrightarrow \infty$ all candidate paths have the same probability of being chosen, i.e., the process approaches a uniform random distribution. When $\mathrm{T} ! 0$, the path with less weight has a high probability of being chosen.

$F\left(R_{j}\right)$ is the path chosen $\left(F\left(R_{j} \in R_{j}\right)\right.$, the choice is made according to equation 4 :

$$
\left(F\left(R_{j}\right)=\max \left\{X \times Q_{j}^{i}\left(\gamma_{j}^{i}\right) \mid \forall \gamma_{j}^{i} \in R_{j} X \approx \cup([0,1])\right\}\right.
$$

After chosing the path, it is checked whether the last segment of the path the vehicle $\mathrm{V}$ is in range of the roadside controller is its ultimate destination (line 9). If it is not like thet, then this is the last segment of the path in the area of that RSU (line 10). The remaining stretches of road are added to the new Vehicle (lines 11-12). However, if the last segment of the path the vehicle V in the range of the RSU is the ultimate destination, then it is the end of the route (line 15).

\section{Results}

This section presents the results of using the proposed system compared to the traditional approach where TC is not automated and does not interact with RSU, but the system does not have an alternate routing. In Fig. 1 shows the results obtained by the system. As can be seen from the figure, the proposed system reduces the effects, caused by congestion (increased travel time, fuel consumption and emissions $\mathrm{O}_{2}$ ). Figure 1 (a) shows that the proposed system reduces the travel time by $23 \%$ in comparison with the traditional approach. This confirms the efficiency of the algorithm of alternative routing. Figure 1(b) shows that the proposed system reduces fuel consumption by $9 \%$ compared to the traditional approach. This reduction is due to the fact that the proposed system detects and eliminates congestion by using alternative paths to avoid congested areas. The proposed mechanism for congestion control that contributes to more effective and continuous movement that leads to a reduction in fuel consumption. In addition, it reduces braking and acceleration, which also contributes to increased fuel consumption. 
Consuming less fuel, less $\mathrm{O}_{2}$ is emitted, as we can observe in figure 1 (C). The proposed system introduces a reduction of up to approximately $10 \%$ in emissions compared to the traditional approach.

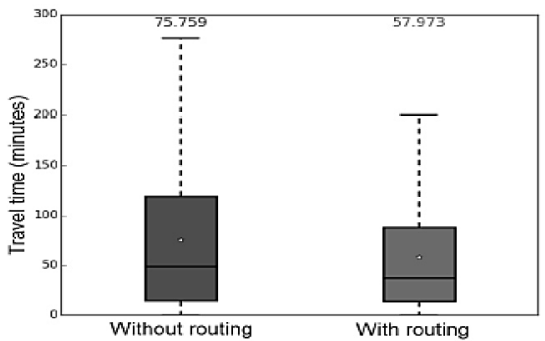

(a) Travel time

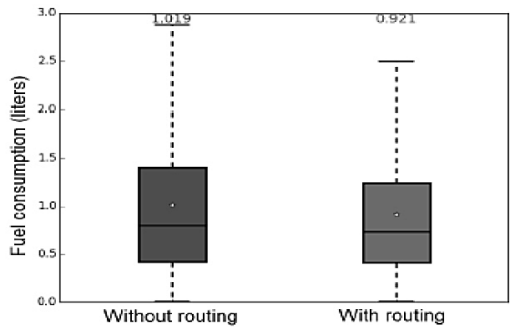

(b) Fuel consumption

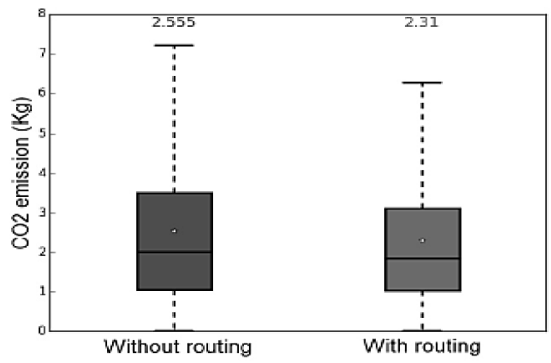

(c) $\mathrm{CO} 2$ emission

Fig. 1. The results of modelling
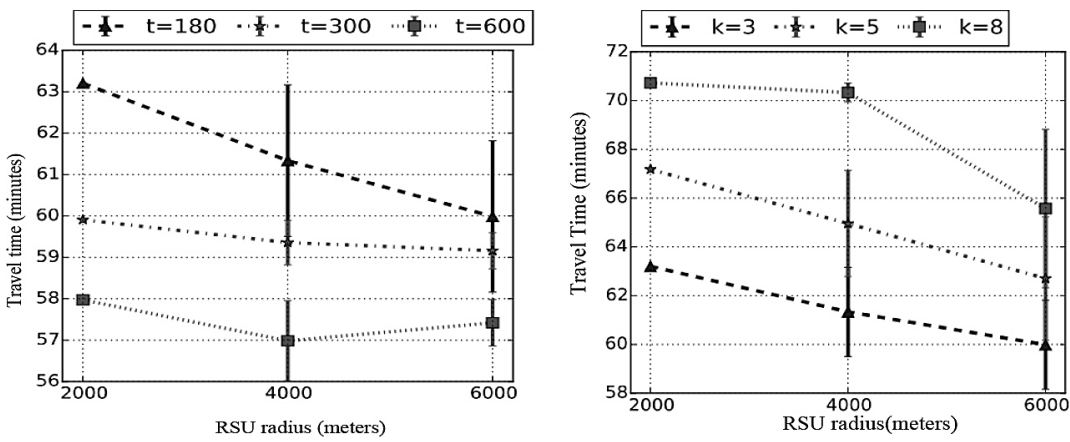

Fig. 2. Diagram of the results of travel time with the change of radius of communication RSU 
Figure 2 shows the average travel time when changing the radius of communication in RSU (2, 4 and $6 \mathrm{~km})$, the path to the shortest (3, 5 and 8$)$ and the frequency of re-routing (180, 360 and 600 seconds). Figure 2 (a) shows the results of the K shortest paths with a fixed frequency of re-route in 180 seconds and varying the communication range of RSU from 2; 4 and $6 \mathrm{~km}$. As we can observe, the higher communication range of RSUs, the larger the reduction in travel time. This is because RSUs have a greater knowledge of the area of the map and the calculated path is better from the standpoint of the time when calculated for a smaller area of the map. In addition, we can observe that the number of $\mathrm{K}$ shortest paths has an impact on the results obtained using the proposed system. For varying $K$, we can note that $K=3$ has better performance compared to other values for $\mathrm{K}$, thus , the result will be reduced to $9 \%$ against $\mathrm{k}=8$ and the reduction to $4 \%$ to $=5$. Figure 2 (b) shows the results with frequency of re-routing $180 ; 360$ and 600 seconds, and $\mathrm{K}$ shortest paths recorded in 3 (higher performance shown in the comparison figure 2 (a)) and the different range of communication from RSUs 2; 4 and $6 \mathrm{~km}$. As we can observe, the frequency of re-routing has a positive effect on reducing the average travel time in the proposed system. The results show that the more the frequency of re-routing, the greater the reduction in the average time of passage for the proposed system. Figure 3 shows the fuel consumption of the proposed system by changing the communication range of RSU in $(2,4$ and $6 \mathrm{~km}$ ), $\mathrm{K}$ shortest paths (3,5 and 8), and the frequency of re-routing $(180 ; 360$ and 600 seconds). Figure 3 (a) shows the results of the $\mathrm{K}$ shortest paths ( $3 ; 5$ and 8 ) with the frequency of re-route fixed in 180 seconds and varying the communication range of RSUs from 2, 4 and $6 \mathrm{~km}$. As we can observe, the higher communication range of the RSU, the greater the reduction in fuel consumption. This is because the RSU have a greater knowledge of the area maps and design way better when calculated for the smaller area of the map. In addition, it is possible to notice that the value of $\mathrm{K}$ shortest paths has an impact on the results obtained using the proposed system. The results show the difference in the amount of up to $7 \%$ for the evaluated configurations. 

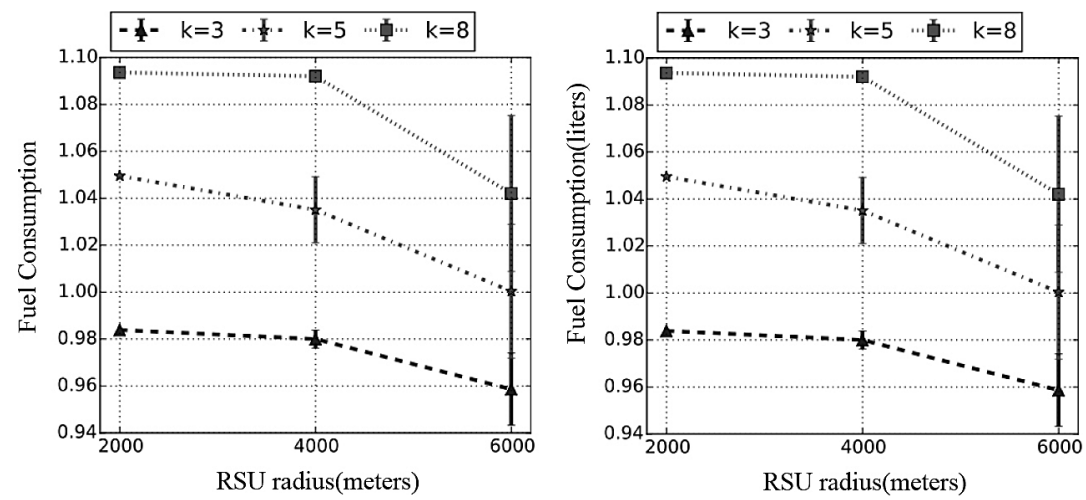

Fig. 3. Diagram of the results of fuel consumption

Consuming less fuel, the vehicle produces less emissions, which is shown in figure 3 . The proposed system provides a reduction of approximately $10 \%$ of emissions compared to the traditional approach. Figure 3 (b) shows the results with frequency of rerouting 180, 360 and 600 seconds in the presence of K shortest paths (figure 3 (a)) and the different range of communication from RSU 2, 4 and $6 \mathrm{~km}$ (figure 3(b)). As we can observe, the range of re-routing positively contributes to reducing fuel consumption in the proposed system. The results show a difference of up to $4 \%$ in relation to the intervals of re-routing from 180 to 600 seconds. The results are presented in figures 2, 3 and 4 show that the higher the communication range of RSUs, the larger the reduction in travel time, fuel consumption and $\mathrm{O}_{2}$ emissions. The obtained results allow to conclude that the smaller the number $\mathrm{K}$ shortest paths, the smaller the effect caused by congestion management. We note that large values of $\mathrm{K}$ also directly affect travel time, fuel consumption and $\mathrm{O}_{2}$ emissions. Another important factor is the frequency of re-routing: the longer the interval, the more reduced travel time, fuel consumption and $\mathrm{O}_{2}$ emissions. In this case, the vehicle does not need to make unnecessary changes of the path, thereby causing unnecessary braking and acceleration, because of high frequency of re-routing contributes to a more efficient and continuous movement, as you can see in figures 2 (b), 3 (b) and 4 (b). 
The results obtained using the proposed system for $\mathrm{O}_{2}$ emissions presented in figure 4 , when changing the communication range of RSU $(2,4$ and $6 \mathrm{~km}$ ), in the presence of $\mathrm{K}$ shortest routes (3,5 and 8) and the frequency of re-routing (180,360 and 600 seconds). Emissions associated with fuel consumption are shown in figures 4 (a) and 4 (b) are the result of fuel consumption. Emissions associated with fuel consumption are shown in figures 4 (a) and 4 (b) are the result of fuel consumption, graphs of which are depicted in figures 3 (a) and 3 (b). The results are presented in figures 2 , 3 and 4 show that the higher the communication range of RSUs, the larger the reduction in travel time, fuel consumption and $\mathrm{O}_{2}$ emissions.
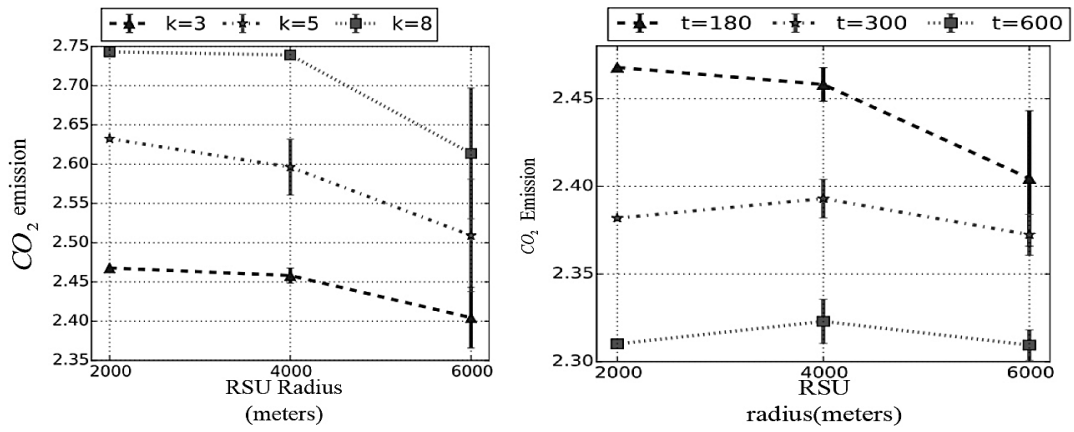

Fig. 4. Diagram of the results of $\mathrm{O}_{2}$ emission

\section{Conclusion}

In this work, the algorithms for traffic management in intelligent transportation system for use in metropolitan areas. The proposed algorithms allow to reduce travel time, fuel consumption and emissions. The results show that the proposed system can significantly reduce average travel time, fuel consumption and emissions .

This work is aimed at implementing a distributed intelligent transport system for detection and control of overloads. For this purpose, RSUs are distributed throughout the city to provide full coverage of the region. In addition, each RSU is responsible for managing congestion only in the area covered by its range. Thus, the vehicle can interact with a number of shares of the way to reducing the harmful impact of an overload, such as increased travel time, fuel consumption and $\mathrm{O}_{2}$ emissions. 


\section{References}

1. Wardrop J.G. Some theoretical aspects of road traffic research // Proc. Inst. Civil Engineering. II. 1952. 326-379 c.

2. Sheffy Y. Urban Transportation Networks. Englewood Cliffs. N.J: Prentice-Hall, 1985. 416 c.

3. Karagiannis G., Altintas O., Ekici E., Heijenk G., B Jarupan., Lin K., and T Weil. "Vehicular networking: A survey and tutorial on requirements, architectures, challenges, standards and solutions," Communications Surveys Tutorials, IEEE, vol. 13, no. 4, pp. 584-616, Fourth 2011.

4. Villas L.A., Ramos H.S., Boukerche A., Guidoni D.L., Araujo R.B., and Loureiro A.A. "An efficient and robust data dissemination protocol for vehicular ad hoc networks," in Proceedings of the 9th ACM Symposium on Performance Evaluation of Wireless Ad Hoc, Sensor, and Ubiquitous Networks. New York, NY, USA: ACM, 2012, pp. 39-46.

5. Zuidgeest M. (2005). Sustainable Urban Transport Development, A Dynamic Optimisation Approach, TRAIL Thesis Series nr. T2005/3, The Netherlands TRAIL Research School, Delft (NL). 305 c.

6. Fahmy M. and Ranasinghe D. "Discovering automobile congestion and volume using vanet," in ITS Telecommunications, 2008. ITST 2008. $8^{\text {th }}$ International Conference on, Oct 2008, 366-371 c.

7. Maia G., Villas L., Boukerche A., Viana A., Aquino A., and Loureiro A. "Data dissemination in urban vehicular ad hoc networks with diverse traffic conditions," in Computers and Communications (ISCC), 2013 IEEE Symposium on, July 2013, pp. 000 459-000 464.

8. Pan J., Khan M., Sandu Popa I., Zeitouni K. and Borcea C. "Proactive vehicle rerouting strategies for congestion avoidance," in Distributed Computing in Sensor Systems (DCOSS), 2012 IEEE 8th International Conference on, May 2012, pp. 265-272.

9. Doolan R. and Muntean G.-M. "Vanet-enabled eco-friendly road characteristics aware routing for vehicular traffic," in Vehicular Technology Conference (VTC Spring), 2013 IEEE 77th, June 2013, pp. 1-5.

10.Zhankaziev S.V. Project development of intelligent transport systems in 2016 MADI. 107. 
11. Uppoor S. and Fiore M. "Large-scale urban vehicular mobility for networking research," in Vehicular Networking Conference (VNC), 2011 IEEE. IEEE, 2011, pp. 62-69.

\section{DATA ABOUT THE AUTHORS}

Nikolaev Andrey Borisovich, Honoris Causa, Doctor of Technical Sciences, Professor, Head of Department

State Technical University - MADI

64, Leningradsky prospekt, Moscow, 125319, Russian Federation nikolaev.madi@mail.ru

Aung Myo Thiwn, Postgraduate Student, Department of Automated Control Systems

State Technical University - MADI

64, Leningradsky prospekt, Moscow, 125319, Russian Federation aungmyothwin252@gmail.com

Myo Lin Aung, Postgraduate Student, Department of Automated Control Systems

State Technical University - MADI

64, Leningradsky prospekt, Moscow, 125319, Russian Federation 109miketee110@gmail.com

Myo Min Khaing, Postgraduate Student, Department of Automated Control Systems

State Technical University - MADI

64, Leningradsky prospekt, Moscow, 125319, Russian Federation myominkhain52@gmail.com

Aung Nyi Nyi Zaw, Postgraduate Student, Department of Automated Control Systems

State Technical University - MADI

64, Leningradsky prospekt, Moscow, 125319, Russian Federation aungnyinyizaw52@gmail.com 